\title{
Quenching of Chlorophyll Fluorescence Induced by Silver
}

\section{Nanoparticles}

\begin{abstract}
A.M. Queiroz ${ }^{\mathrm{a}, \mathrm{b}}$, A.V. Mezacasa ${ }^{\mathrm{b}}$, D.E. Graciano ${ }^{\mathrm{b}}$, W.F. Falco ${ }^{\mathrm{b}}$, J.-C.M'Peko ${ }^{\mathrm{c}}$, F.E.G. Guimarães $^{\mathrm{c}}$, T. Lawson ${ }^{\mathrm{d}}$, I. Colbeck ${ }^{\mathrm{d}}$, S.L. Oliveira ${ }^{\mathrm{a}}$, A.R.L. Caires ${ }^{\mathrm{a}, *}$

a Grupo de Óptica e Fotônica, Instituto de Física, Universidade Federal de Mato Grosso do Sul, CP 549, 79070-900 Campo Grande, MS, Brazil

b Grupo de Óptica Aplicada, Universidade Federal da Grande Dourados, CP 533, 79804-970 Dourados, MS, Brazil

c Instituto de Física de São Carlos, Universidade de São Paulo, CP 369, 13560-970 São Carlos, SP, Brazil

d School of Biological Sciences, University of Essex, Wivenhoe Park, CO4 3SQ Colchester, Essex, United Kingdom
\end{abstract}

\begin{abstract}
The interaction between chlorophyll (Chl) and silver nanoparticles (AgNPs) was evaluated by analyzing the optical behavior of Chl molecules surrounded by different concentrations of AgNPs (10, 60, and $100 \mathrm{~nm}$ of diameter). UV-Vis absorption, steady state and time-resolved fluorescence measurements were performed for $\mathrm{Chl}$ in the presence and absence of these nanoparticles. AgNPs strongly suppressed the Chl fluorescence intensity at $678 \mathrm{~nm}$. The Stern-Volmer constant $\left(\mathrm{K}_{\mathrm{SV}}\right)$ showed that fluorescence suppression is driven by the dynamic quenching process. In particular, $\mathrm{K}_{\mathrm{SV}}$ was nanoparticle size-dependent with a nearly linear decrease as a function of the nanoparticle diameter. Finally, changes in the Chl fluorescence lifetime in the presence of nanoparticles demonstrated that the fluorescence quenching may be induced by the excited electron transfer from the $\mathrm{Chl}$ molecules to the metal nanoparticles.
\end{abstract}

Keywords: Chlorophyll; Fluorescence; Lifetime; Quenching; Silver; Nanoparticle.

*Correspondent author. E-mail: anderson.caires@ufms.br. 


\section{Introduction}

The development of nanotechnologies in recent decades has led to an indiscriminate use of nanomaterials (NMs) in various industrial applications [1]. The concern about the impact of nanoparticles (NPs) in the environment has increased because their release and accumulation are inevitable [2]. NMs may constitute a toxicological risk as nanosized particles have presented toxicity in a variety of organisms, being generally more toxic than larger particles (bulk) [3]. In fact, NPs behave differently from bulk materials, with regard to chemical, physical and biological properties, due to a change in the nature of the interaction forces between NPs and environment. Consequently, the size of the NPs is a key feature in relation to the effects that NPs can induce in the environment, human health and biosphere as a whole [4].

Silver nanoparticles (AgNPs) are among the most used NPs for developing new technologies and commercial products due to their antimicrobial properties [7]. The wide variety of commercial applications of the AgNPs results in a significant increase in their production and, consequently, release to the environment [8,9]. Plants are particularly relevant in this scenario because of their constant interaction with the air, soil, and water so that they are potentially exposed to NPs. In addition, plants may represent a possible agent for NPs' bioaccumulation and biomagnification due to their importance in the food chain since they are consumed by organisms of different trophic levels $[5,6]$.

Recently, several studies about the impacts of AgNPs on plant development were reported. For instance, Ma et al. showed that AgNPs are toxic to seedlings of watercress even at low concentrations $(<1 \mathrm{ppm})[6]$. They observed that different concentrations of AgNPs with diameter between 20 and $80 \mathrm{~nm}$ affected plant growth and the nanoparticle phytotoxicity depends on the size and concentration of the AgNPs. In turn, Jiang et al. 
also demonstrated that AgNPs induced a significant decrease in the plant biomass, chlorophyll content and photosynthetic efficiency of photosystem II (Fv/Fm) in Spirodela polyrhiza [10].

During photosynthesis, when light energy is converted into chemical energy by plants, the key role is played by chlorophyll $a$ because it absorbs and transfers energy to the reaction centers, inducing charge separation and, subsequently, photosynthetic electron transport [11]. In addition, it is well established that chlorophyll fluorescence (ChlF) may be applied to evaluate the physiological status of plants, in vivo, [12-13] as chlorophyll molecule is an intrinsic fluorophore present in green plants. Consequently, ChlF can been used for monitoring, directly or indirectly, the environmental impact induced by biotic or abiotic agents as the photosynthetic efficiency of plants usually decreases when placed under a stress condition [14-17].

To obtain fundamental knowledge about interaction between Chl molecules and AgNPs and to evaluate the potential application of ChlF spectroscopy as an analytical technique for further investigation about nanoparticle effects on plants, the present study performed a close analysis of ChlF behavior, extracted from leaves of fava bean (Vicia faba L.), when surrounded by different diameters and concentrations of AgNPs.

\section{Materials and methods}

\subsection{Silver nanoparticles}

Silver nanoparticles (AgNPs) with spherical form at $0.02 \mathrm{mg} \cdot \mathrm{mL}^{-1}(185 \mu \mathrm{M})$ in aqueous buffer, containing sodium citrate as stabilizer, were purchased from SigmaAldrich (Brazil). As informed by the company, the nanoparticle diameters were determined by transmission electron microscopy (TEM) and the mean diameters of the 
used $\mathrm{AgNPs}$ were $100 \pm 8,60 \pm 8$, and $10 \pm 4 \mathrm{~nm}$. Additional information of the AgNPs is presented in the Supplementary Material (Fig. S1).

In all analyses performed, the AgNPs were suspended in a dilute aqueous citrate buffer for stabilizing the nanoparticles and to prevent aggregation. This buffer solution was selected because the citrate-based agent is weakly bound with the nanoparticle surface and can be readily replaced by other molecule, allowing a direct interaction between chlorophyll molecules and AgNPs surface.

\subsection{Chlorophyll extract}

Leaves of fava bean (Vicia faba L.) were collected and cut into small pieces. $30 \mathrm{~mL}$ of methanol PA was added for each $3 \mathrm{~g}$ of leaves and they were incubated for $72 \mathrm{~h}$ at 2 ${ }^{\circ} \mathrm{C}$. Then, chlorophyll extract was separated and stored in amber bottle at around $2{ }^{\circ} \mathrm{C}$. The chlorophyll content was determined by using the Arnon method adapted by Porra (Eq. 1) [18], where $\mathrm{A}^{652.0}\left(\mathrm{~A}^{665.2}\right)$ is the absorbance value at $652.0 \mathrm{~nm}(665.2 \mathrm{~nm})$ and 24.23 (3.26) obtained from the millimolar extinction coefficients of chlorophylls at $652.0 \mathrm{~nm}(665.2 \mathrm{~nm})$ for simultaneous determination of the total chlorophyll content $[C h l a+b]$, in $\mu \mathrm{M}$ units, in buffered methanol.

$$
[\text { Chl } a+b]=24.23 A^{652.0}+3.26 A^{665.2}
$$

\subsection{Nanoparticle-chlorophyll solution}

To study the interaction between chlorophyll and AgNPs, $0.5 \mathrm{~mL}$ of the chlorophyll extract at $14.7 \mu \mathrm{M}$ was added to $2.5 \mathrm{~mL}$ of the AgNPs-containing solution at concentrations of $2.4,9.7,38.6,77.2$, and $154.5 \mu \mathrm{M}$. The same procedure was carried out for the three nanoparticle sizes $(10,60$, and $100 \mathrm{~nm})$. 


\subsection{Optical analyses}

The molecular absorption in the $200-800 \mathrm{~nm}$ wavelength range were performed in a Cary 50 UV-Vis spectrophotometer (Varian) using a quartz cuvette of $10 \mathrm{~mm}$ optical pathlength at room temperature.

The fluorescence measurements were performed using a fluorimeter consisting of a laser excitation source, a monochromator (2000 USB FL-OceanOptics), a Y-type optical fiber. The fluorescence spectra of the samples were obtained at wavelengths between 450 and $800 \mathrm{~nm}$ with excitation at $405 \mathrm{~nm}$. All measurements were carried out at room temperature using a quartz cuvette with four polished faces and $10 \mathrm{~mm}$ optical pathlength.

Fluorescence lifetime measurements were performed using a multiphoton confocal microscope (Zeiss LSM 780) with a 20x objective and single-photon avalanche diode detector with picosecond temporal resolution. A tunable 690-1100 nm laser (Chameleon, Coherent) was used as the excitation source. Lifetime measurements of the samples were taken at $678 \mathrm{~nm}$ with a two-photon excitation at $800 \mathrm{~nm}$. The excitation laser provides 140-fs pulses with a repetition rate of $80 \mathrm{MHz}$.

\section{Results and Discussion}

Fig. 1 shows the absorption and fluorescence spectra for the Chl extract in the absence of AgNPs. The Soret and $\mathrm{Q}_{\mathrm{x}, \mathrm{y}}$ absorption bands were observed in the 400-450 and 600-750 $\mathrm{nm}$ wavelength ranges, respectively (Fig. 1a). In turn, a prominent emission band centered at $678 \mathrm{~nm}$ was observed along with a weak shoulder located around $728 \mathrm{~nm}$ (Fig. 1b) [19].

Absorption data of AgNPs not surrounded by Chl molecules were also collected. For instance, the absorption spectrum of the AgNPs of $60 \mathrm{~nm}$ is presented in Fig. 2a. An 
absorption band at around $430 \mathrm{~nm}$ due to the plasmon resonance induced by the collective oscillation of the electrons on the metal surface was observed [20]. Additionally, the UV-Vis absorption analysis revealed that the plasmon resonance is suppressed whether the $\mathrm{pH}$ of the solution is acid or basic, as presented in the Fig. S2 in the supplementary materials. This result is possibly due to the formation of nanoparticle aggregation as $\mathrm{pH}$ is one of the most important factors in the formation of nanoparticle clusters [21].

Considering the previous finding, absorption spectra of the solutions with neutral $\mathrm{pH}$ containing AgNPs with different nanoparticle sizes and concentrations were acquired (data not show). Fig. 2b highlights the linear red-shift of the absorption peak of the AgNPs solution with increasing nanoparticle diameter (see the full spectra in Fig. S1 in the supplementary materials). Similar results for metal nanoparticles were also verified elsewhere [22] as the absorption maximum of plasmon resonance shifts to higher wavelengths as nanoparticle size increase [22].

Fig. 2c displays that the plasmon absorption intensity linearly increased as a function of the AgNPs concentration, with $\mathrm{R}^{2}$ coefficients of $0.9912,0.9997$, and 0.9972 for the diameter of 10,60 , and $100 \mathrm{~nm}$, respectively. Besides, the angular coefficient obtained from Fig. 2c linearly decreases as a function of nanoparticle size presenting a $\mathrm{R}^{2}$ coefficient of 0.9968 (Fig. 2d). In fact, these results may be accounted by the metal surface area dependence of the plasmon resonance [22]. For instance, it is known that the total metal surface area increases as nanoparticle concentration increases, enhancing of the plasmon resonance absorption (Fig. 2c) as well as the total metal surface area decreases as nanoparticle size increases, for a given nanoparticle concentration, reducing the plasmon resonance absorption (Fig. 2d) [22]. 
The chlorophyll-AgNPs interaction was studied based on the effect of the nanoparticle size and concentration. Solutions with neutral $\mathrm{pH}$ containing $\mathrm{Chl}$ at about 2.4 $\mu \mathrm{M}$ and AgNPs with different nanoparticle sizes and concentrations were used. The $\mathrm{UV}-\mathrm{V}$ is absorption results revealed that no additional band as well as no band shift of both chlorophyll and plasmon absorption was observed in the chlorophyll-AgNPs solutions (Fig. S3 and S4 of the supplementary materials). It was verified only that the spectra are composed by the resultant of the individual spectra. Therefore, these results indicate that there is no Chl-AgNPs hybrid complex formation in the ground state.

The fluorescence spectra of the chlorophyll-AgNPs solutions in the 625-800 nm spectra range with excitation at $405 \mathrm{~nm}$ were obtained and the results revealed that $\mathrm{ChlF}$ was suppressed as a function of AgNPs, as shown in Fig. S5 in the supplementary materials. Fig. 3a summarizes the $\mathrm{F}_{0} / \mathrm{F}$ fluorescence ratio for different diameters and AgNPs concentration, where $\mathrm{F}_{0}$ and $\mathrm{F}$ are the ChlF intensity at $678 \mathrm{~nm}$ in the absence and presence of the silver nanoparticles, respectively. The fluorescence suppression was linear as a function of nanoparticle concentration, presenting a $\mathrm{R}^{2}$ coefficient of 0.9854 , 0.9459 , and 0.9413 for the nanoparticle diameter of 10, 60 and $100 \mathrm{~nm}$, respectively, as well as it relies on the diameter of the AgNPs. Based on these results, the following Stern-Volmer equation describes the observed Chl suppression:

$$
\frac{F_{o}}{F}=1+K_{S V}[Q]
$$

where $K_{S V}$ is the Stern-Volmer quenching constant and $Q$ is the concentration of the suppressor agent (AgNPs) [19]. This remark agrees with the discussion reported by Falco et al. $[19,23]$, in which chlorophyll molecules may be adsorbed on the metal nanoparticle surface resulting in a quenching of ChlF. For instance, our data fit provided a $K_{S V}$ of $3.10^{3} \mathrm{M}^{-1}$ for the nanoparticle of $100 \mathrm{~nm}$. Similar value $\left(1.10^{3} \mathrm{M}^{-1}\right)$ was 
reported by Falco et al. as a result of the interaction between Chl and AgNPs of $100 \mathrm{~nm}$ [23].

The distinct angular coefficient of $\mathrm{F}_{0} / \mathrm{F}$ versus $\mathrm{AgNPs}$ content for the three nanoparticle sizes is an indicative that $K_{S V}$ is size-dependent. The values estimated for $K_{S V}$ are illustrated in Fig. 3b. An exponential decay relationship between the $K_{S V}$ and AgNPs diameter, governed by Eq. 2 with $\mathrm{R}^{2}$ of 0.9999, is exhibited in Fig. $3 b$.

$$
K_{S V}=0.53+24.4 e^{-\left(\frac{S}{43.6}\right)}
$$

where $S$ is the nanoparticle size.

The linear fluorescence suppression as a function of the suppressor concentration may be induced by either a static or a dynamic quenching process [24]. Fig. 4a shows the fluorescence decays related to the ChlF for different nanoparticle concentration with diameter of $10 \mathrm{~nm}$. The curves were obtained monitoring the $\mathrm{ChlF}$ at $678 \mathrm{~nm}$ under two-photon excitation at $800 \mathrm{~nm}$. The fluorescence decays were fitted to the following second order exponential decay equation:

$$
F(t)=\sum_{i}^{2} A_{i} e^{\left[-\tau / \tau_{i}\right]}
$$

where $F$ is the fluorescence intensity, $A_{i}$ are the amplitudes (pre-exponential factors), $t$ is the time, and $\tau_{i}$ is the fluorescence lifetimes [23]. The lifetime data indicate that the quenching process must be of dynamic type as in the static quenching the lifetime is not expected to be altered by the suppressor [24]. Furthermore, a close analysis of the lifetime-dependence with Chlorophyll concentration in Fig. 4b demonstrates that the interaction between chlorophyll molecules and silver nanoparticles occurs mainly during the first nanosecond (below $0.20 \mathrm{~ns}$ ) after the excitation pulse input. The short lifetime $\left(\tau_{1}\right)$ linearly decreases with increasing 
nanoparticle concentration, revealing that the physicochemical mechanism responsible for the lifetime reduction may be directly related to the increase of the metal surface area in the solution.

It is worth to point out that the two major mechanisms responsible to fluorescence quenching induced by metal nanoparticles are the energy-transfer and the electron-transfer [25]. The small overlap between AgNPs absorption and ChlF corroborates with the statement that the ChlF suppression should be mostly attributed to the electron transfer from Chl to AgNPs. Additionally, as no Chl-AgNPs hybrid complex formation was observed in the ground state, the results also indicate that ChlF quenching occurred by the electron transfer from excited Chl to AgNPs. In fact, direct binding of a fluorophore to the metal surface in general results in the quenching of excited states [26]. Furthermore, by analyzing the interaction between Chl molecules and gold nanoparticles (AuNPs), Barazzouk and co-workers has also demonstrated that the main suppression process of the ChlF was induced by the photoinduced electron transfer from excited Chl to AuNPs [27].

Based on that, the excited electron transfer from the $\mathrm{Chl}$ molecules to the metal surface after the excitation process. Therefore, in the presence of nanoparticles-related metal surfaces, the excited state may be deactivated by an additional decay channel via a non-radiative transition (electron transfer) instead of light emission (fluorescence), leading to a decrease in lifetime $\tau_{1}$ and fluorescence intensity in agreement with the steady state fluorescence data (Fig. 3b). Fig. 4c presents that long lifetime $\left(\tau_{2}\right)$ remains practically constant $(3.68 \pm 0.03)$ ns with increasing nanoparticle concentration, except for a decrease experienced at the highest concentration $(\sim 154.5 \mu \mathrm{M})$.

In summary, the results demonstrate that the lifetime dependence of the fluorescence is strongly affected when fast nonradiative processes are competing with 
the relatively slow radiative process of about $3.6 \mathrm{~ns}$. In the presence of silver nanoparticles, the fluorescence emission is strongly quenched in the time scale of 1001000 ps when compared with chlorophyll solution without AgNPs(Fig. 4a). The total fluorescence decay has contributions from the emission of non-interacting chlorophyll in the supernatant solution and chlorophyll interacting with the nanoparticles. The increase of nanoparticle content in the solution reduces the concentration of free chlorophyll through interaction with the metal surface. Thus, the strong reduction of the short fluorescence decay time $\left(\tau_{1}\right)$ with nanoparticle concentration is associated to the enhanced quenching process - which are faster than the radiative one - due to charge transfer from the excited chlorophyll states to resonant silver states.

Fig. 4 refers to data obtained for the experiments involving AgNPs with $10 \mathrm{~nm}$ of diameter; the behavior was qualitatively similar for 60 and $100 \mathrm{~nm}$ (data not shown). The estimated values of $\mathrm{Chl}$ lifetime fluorescence for all the considered AgNPs sizes are depicted in Fig. 5. Because revealing also nanoparticle size-dependent, these results confirm that only the short lifetime is affected by the interaction between chlorophyll and metal surface. In fact, it was determined a linear nanoparticle size-dependence of $\tau_{1}$, for any given $\left(2.41 \mu \mathrm{M}\right.$ in this figure) nanoparticle concentration, while $\tau_{2}$ was not nanoparticle size-sensitive.

\section{Conclusions}

Optical measurements can be used for monitoring the interaction between $\mathrm{Chl}$ and AgNPs. It was demonstrated that AgNPs quenched the ChlF and contributed for the reduction in the ChlF lifetime. Besides, the resulting Chl-AgNPs interaction is dependent on size and concentration of the metal nanoparticles. The results also reveal 
that fluorescence suppression is caused by a dynamic quenching process and the SternVolmer constant $\left(\mathrm{K}_{\mathrm{SV}}\right)$ is linearly nanoparticle size-dependent. This optical behavior is indicative that $\mathrm{Chl}$ molecules may be adsorbed at the metallic AgNPs surfaces and then the excited Chl molecules may transfer their excited electrons to these surfaces, resulting in ChlF quenching and lifetime decrease. In summary, due to the existence of an effective Chl-AgNPs interaction, the findings suggest that AgNPs may represent a potential risk for plants as this interaction may change the operation of photosynthetic apparatus (chemical energy production) of photosynthetic organisms. In this scenario, additional investigations regarding the metal nanoparticles effects on in vivo plants based on steady-state and time-resolved $\mathrm{ChF}$ measurements are still required.

\section{Acknowledgments}

The authors are grateful to CNPq, CAPES and FUNDECT, Brazilian funding agencies, for their financial support. The authors also acknowledge the support provided by the National Institute of Science and Technology of Optics and Photonics (INOF). 


\section{References}

[1] R. J. Aitken, M. Q. Chaudhry, A. B. A. Boxall, M. Hull, Occupational Medicine 56 (2006) 300-306.

[2] P. Landa, R. Vankova, J. Andrlova, J. Hodek, P. Marsik, H. Storchova, J.C. White, T. Vanek, Journal of Hazardous Materials 241 (2012) 55-62.

[3] A. Dhawan, V. Sharma, D. Parmar, Nanotoxicology 3 (2009) 1-9.

[4] H. Bouwmeester, S. Dekkers, M. Y. Noordama, W. I. Hagens, A. S. Bulder, C. Heer, S. E. C. G. Ten Voordea, S. W. P. Wijnhovenb, H. J. P. Marvina, A. J. A. M. Sips, Regulatory Toxicology and Pharmacology 53 (2009) 52-62.

[5] M. A. Maurer-Jones, I. L. Gunsolus, C. J. Murphy, C. L. Haynes, Analytical Chemistry 85 (2013) 3036-3049.

[6] X. Ma, J. Geiser-Lee, Y. Deng, A. Kolmakov, Science of Total Environment 408 (2010) 3053-3061.

[7] S.A. Blaser, M. Scheringer, M. Macleod, K. Hungerbuhler, Science of the Total Environment 390 (2008) 396-409.

[8] F. Gottschalk, T. Sonderer, R. W. Scholz, B. Nowack, Environmental Science \& Technology 43 (2009) 9216-9222.

[9] J. D. Judy, J. M. Unrine, P. M. Bertsch, Environmental Science \& Technology 45 (2011) 776-781.

[10] H. Jiang, M. Li, F. Chang, W. Li, L. Yin, Environmental Toxicology and Chemistry 3 (2012) 1880-1996.

[11] A. B. J. Parusel, S. A Grimme, Journal of Physical Chemistry 104 (2000) 53955398.

[12] N. R. Baker, Annual Review of Plant Biology 59 (2008) 89-113.

[13] A. S. Ndao, A. Konté, M. Biaye, M. E. Faye, N. A. B. Faye, A. Wagué, Journal of Fluorescence 15 (2005) 123-129.

[14] C.-M. Zhao, G.-X. Wang, X.-P. Wei, J.-M. Deng, D.-L. Cheng, Trees-Structure and Function 21 (2007) 55-63.

[15] W. Y. Kao, T. T. Tsai, Plant Cell and Environment 21 (1998) 1055-1062.

[16] Z. G. Cerovic, G. Samson, F. M. Iribas, N. Tremblay, I. Moya, Agronomie 19 (1999) 543-578.

[17] K. Maxwell, G. N. Johnson, Journal of Experimental Botany 51 (2000) 659-668.

[18] R. J. Porra, Photosynthesis Research 73 (2002) 149-156.

[19] W. F. Falco, E. R. Botero, E. A. Falcão, E. F. Santiago, V. S. Bagnato, A. R. L. Caires, Journal of Photochemistry and Photobiology A: Chemistry 225 (2011) 65-71. 
[20] M. A. Farias, M. C. Gonçalves, $34^{\text {a }}$ Reunião Anual da Sociedade Brasileira de Química, São Paulo: 2011.

[21] A. A. Keller, H. T. Wang, D. X. Zhou, H. S. Lenihan, G. Cherr, B. J. Cardinale, R. Miller, Z. X. Ji, Environmental Science \& Technology 44 (2010) 1962-1967.

[22] A. Slistan-Grijalva, R. Herrera-Urbina, J.F. Rivas-Silva, M. Ávalos-Borja, F.F. Castillón-Barraza, A. Posada-Amarillas, Physica E: Low-dimensional Systems and Nanostructures 27 (2005) 104-112.

[23] W. F. Falco, A. M. Queiroz, J. Fernandes, E. R. Botero, E. A. Falcão, F. E. G. Guimarães, J.-C. M'peko, S. L. Oliveira, I. Colbeck, A. R. L. Caires, Journal of Photochemistry and Photobiology A: Chemistry 299 (2015) 203-209.

[24] B. Valeur, Molecular Fluorescence: Principles and Applications, first ed., WileyVCH, Weinheim, 2001.

[25] S. Murphy, L. Huang, P.V. Kamat, Journal of Physical Chemistry C 115 (2011) 22761-22769.

[26] P.V. Kamat, Journal of Physical Chemistry B 106 (2002) 7729-7744.

[27] S. Barazzouk, P. V. Kamat, S. Hotchandani, Journal of Physical Chemistry B 109 (2005) 716-723. 


\section{Figure Captions}

Fig. 1 - (a) Absorption and (b) fluorescence spectrum of chlorophyll extract at 1.15 $\mu \mathrm{M}$. The fluorescence spectrum was obtained by exciting at $405 \mathrm{~nm}$.

Fig. 2 - (a) Absorption spectrum of silver nanoparticles of $60 \mathrm{~nm}$ at $50 \mu \mathrm{M}$ and at $\mathrm{pH} 7$; (b) Plasmon absorption maximum as a function of silver nanoparticle diameter; (c) Plasmon absorption intensity as a function of silver nanoparticle concentration; (d) Angular coefficient related the linear regression shown in Fig $2 \mathrm{c}$ as a function of silver nanoparticle diameter.

Fig. 3 - (a) ChlF ratio $\left(\mathrm{F}_{0} / \mathrm{F}\right)$ at $678 \mathrm{~nm}$ depending on the $\mathrm{AgNPs}$ concentration in $\mathrm{Chl}$ solution at $2.4 \pm 0.4 \mu \mathrm{M}$ under 405 -nm excitation. (b) Stern-Volmer quenching constant (Ksv) versus nanoparticle diameter.

Fig. 4 - (a) Chlorophyll fluorescence decays at $678 \mathrm{~nm}$ for solutions containing different AgNPs concentration with $10 \mathrm{~nm}$ of diameter. The solutions were excited via two photon absorption. (b) Short lifetime $\left(\tau_{1}\right)$ and (c) long lifetime $\left(\tau_{2}\right)$ depending on the nanoparticle concentration. The dashed lines are guides to the eyes.

Fig. 5 - (a) Short and (b) long lifetime of chlorophyll fluorescence in solutions with AgNPs concentration of $2.41 \mu \mathrm{M}$ as a function of the nanoparticle diameter. The lines are guides to the eyes. 

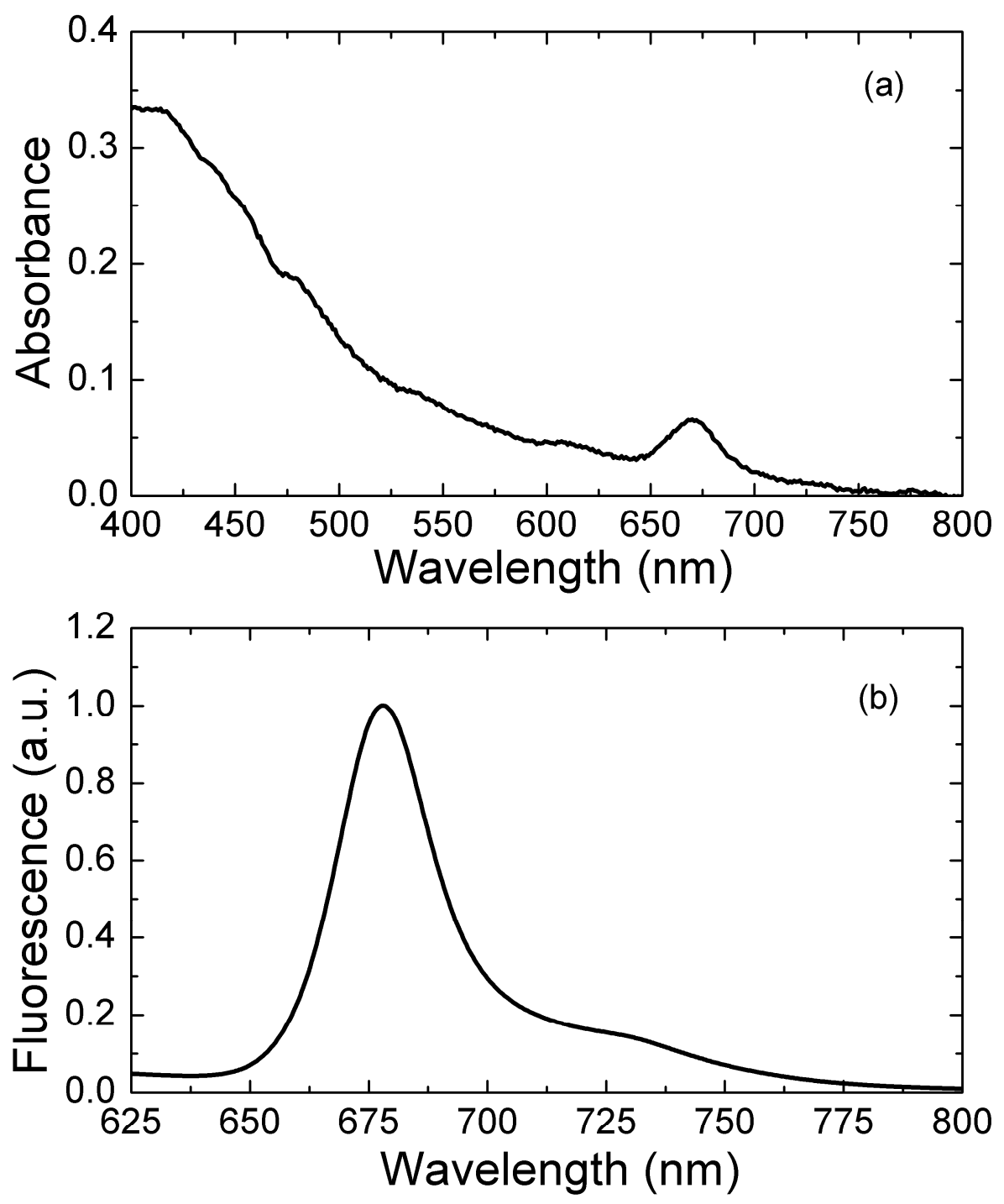

Fig. 1 - A.M. Queiroz et al. 

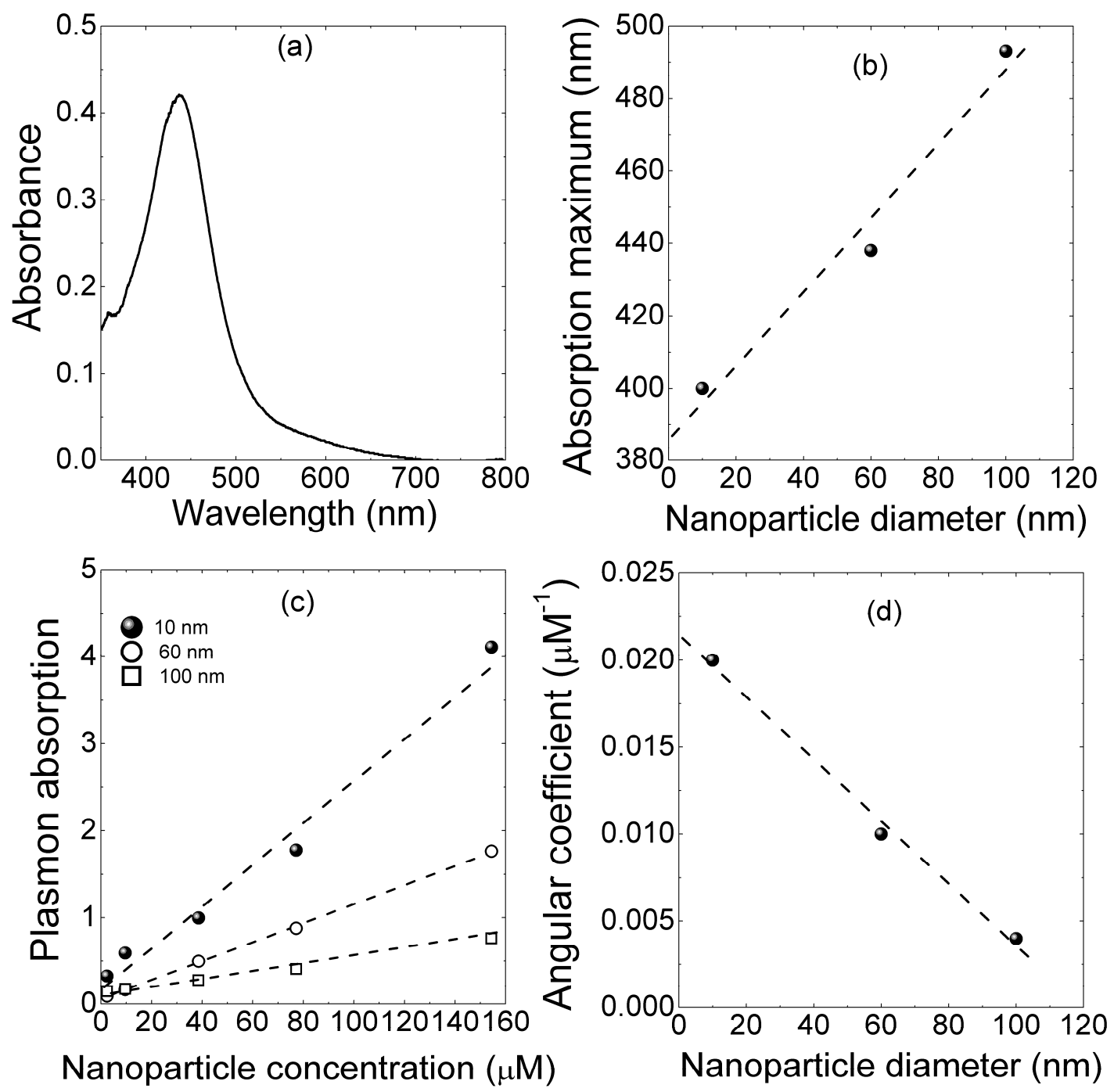

Fig. 2 - A.M. Queiroz et al. 


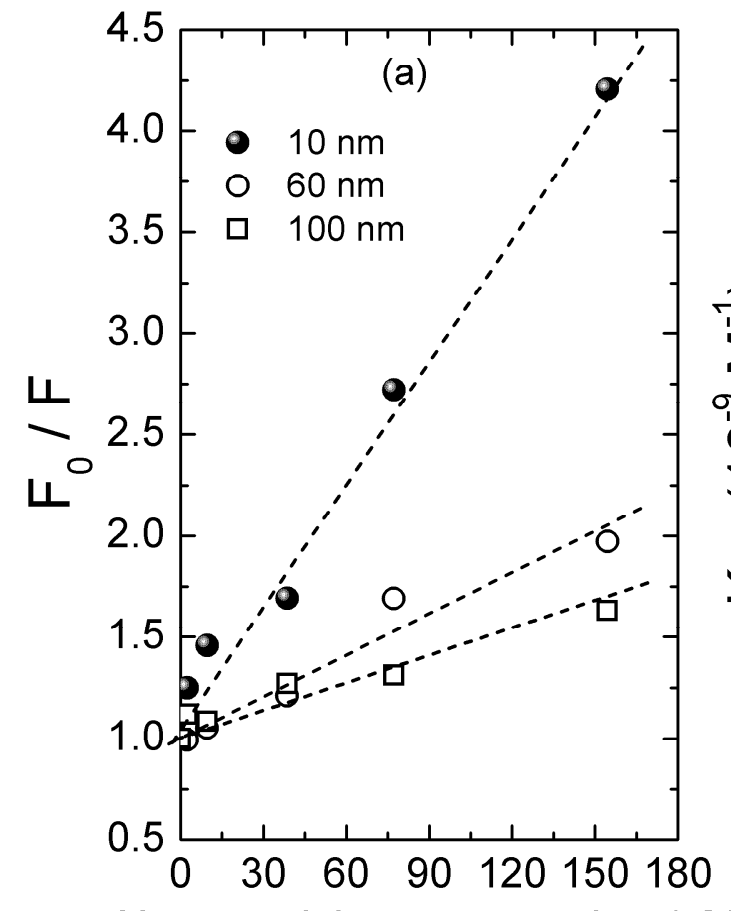

Nanoparticle concentration $(\mu \mathrm{M})$

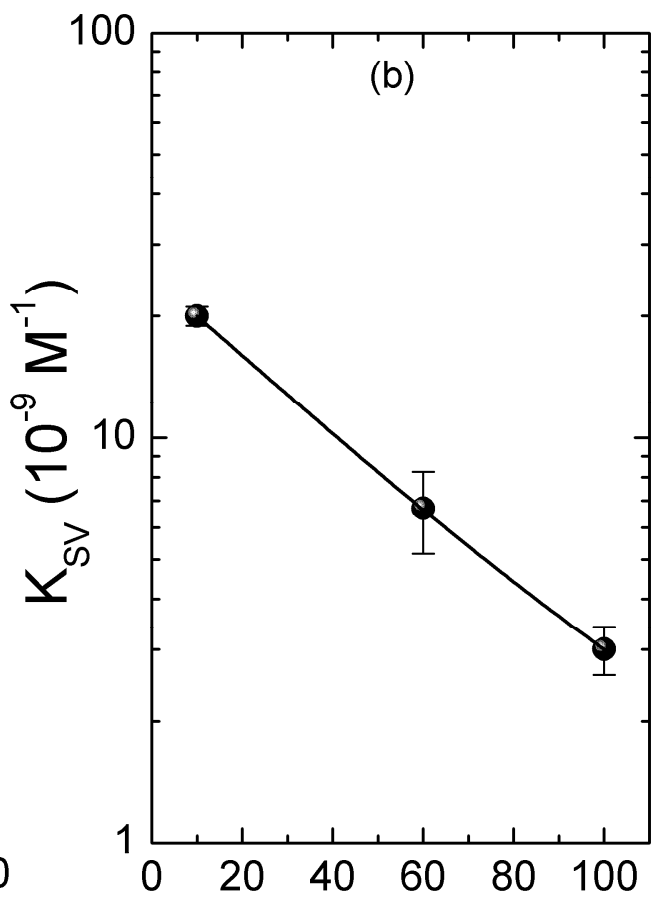

Nanoparticle diameter $(\mathrm{nm})$

Fig. 3 - A.M. Queiroz et al. 

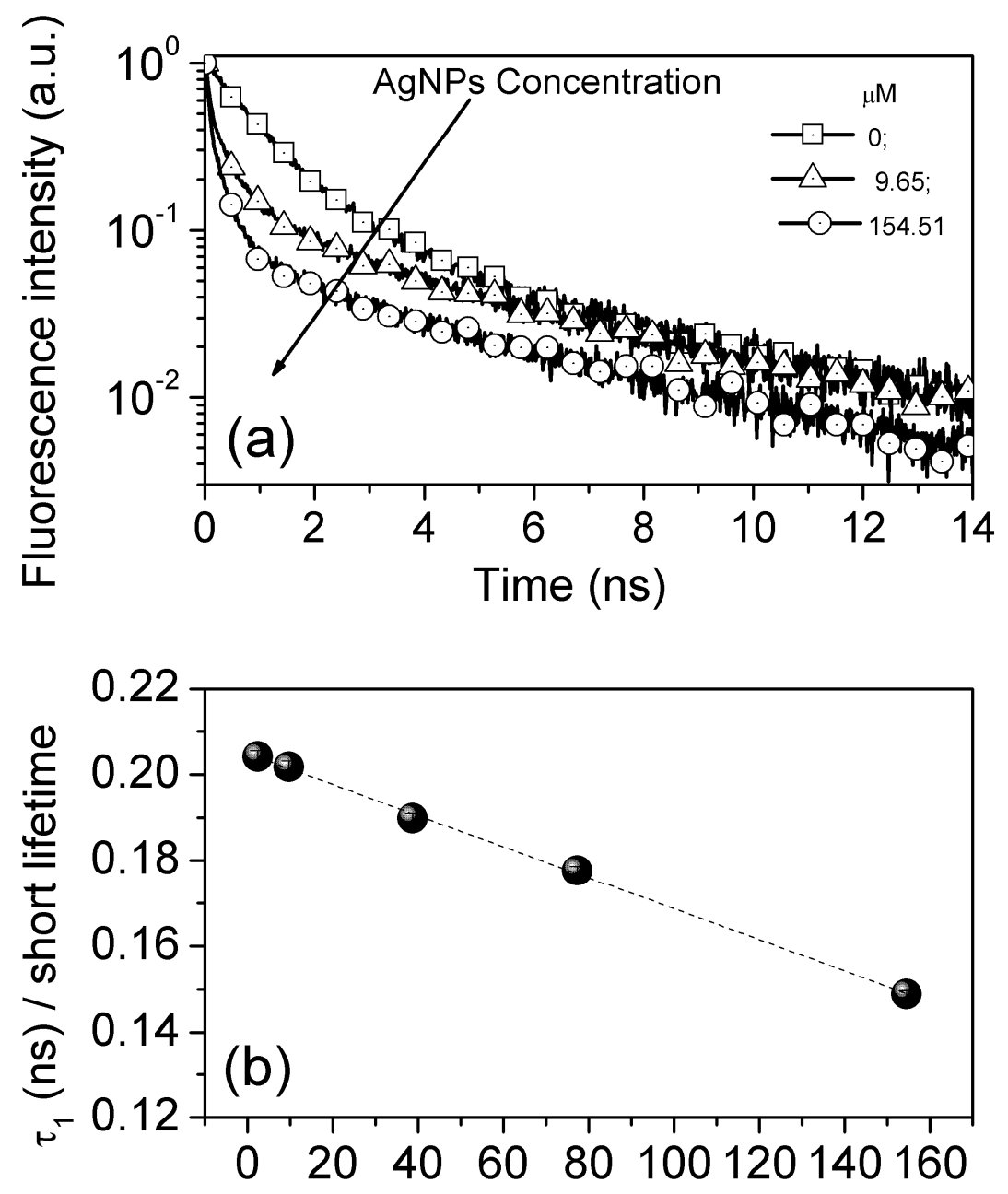

Nanoparticle concentration $(\mu \mathrm{M})$

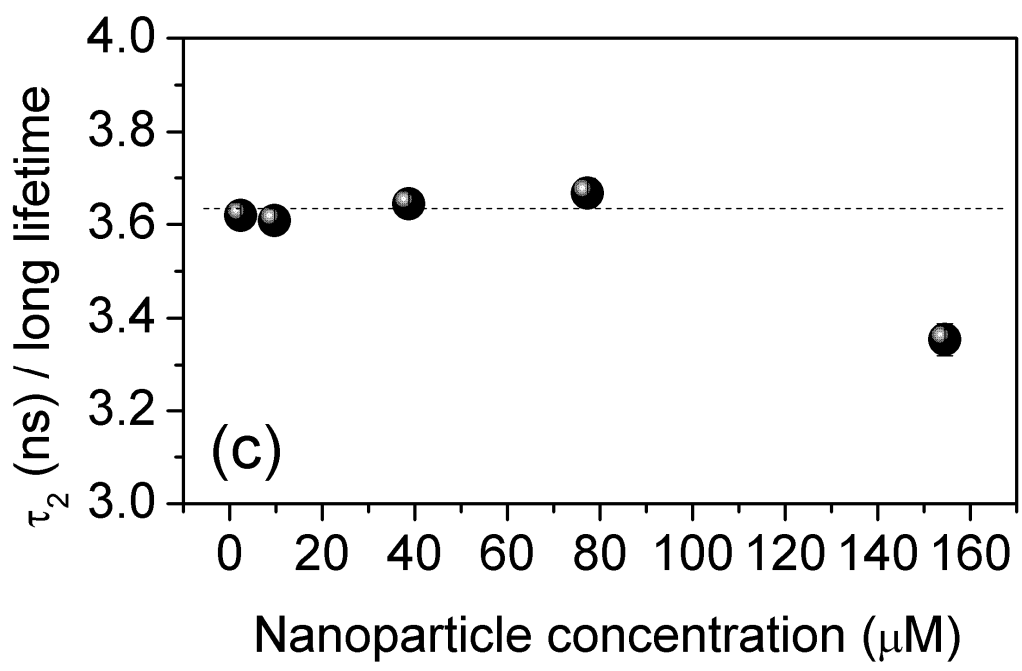

Fig. 4 - A.M. Queiroz et al. 


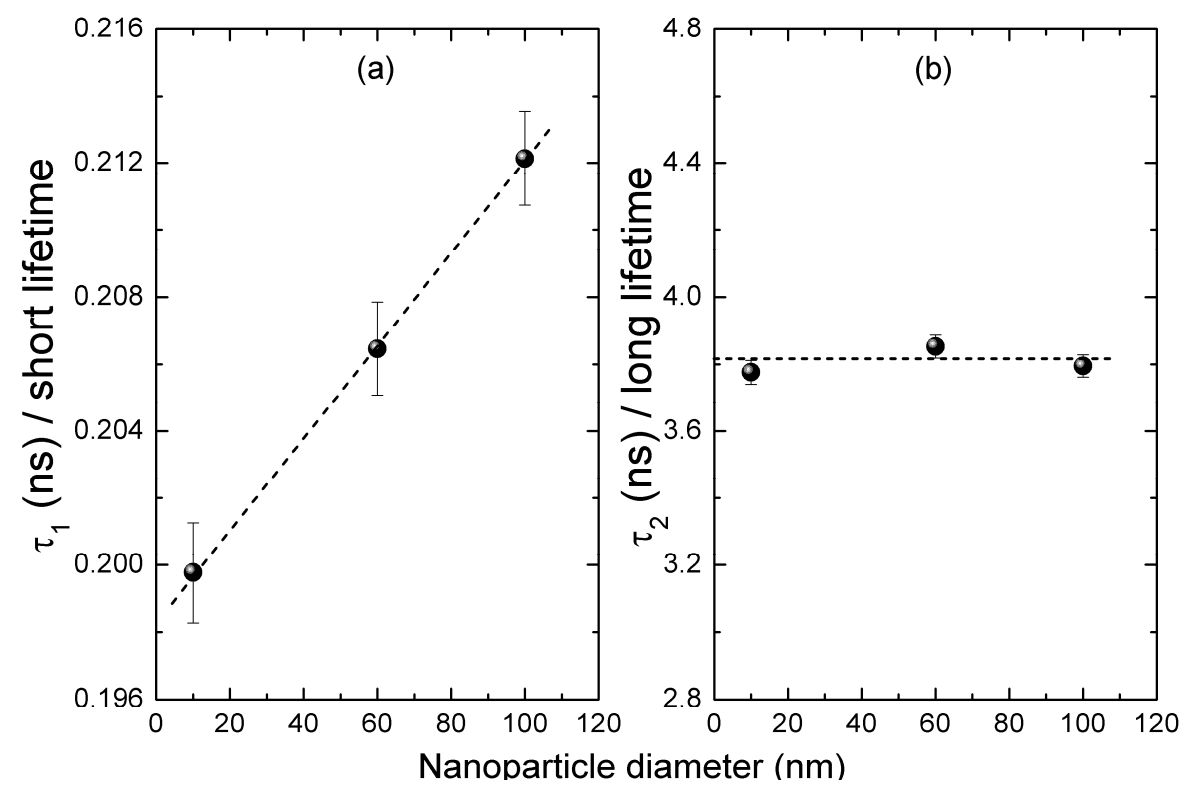

Fig. 5 - A.M. Queiroz et al. 